\title{
Conference summary: triggered star formation in a turbulent ISM
}

\author{
Bruce G. Elmegreen \\ IBM T. J. Watson Research Center, 1101 Kitchawan Road, Yorktown Hts., NY 10598 USA \\ email: bge@us.ibm.com
}

\begin{abstract}
While the overall star formation rate in a galaxy appears to depend primarily on the gas mass and density, with the timescale for conversion of gas into stars given by the dynamical time, turbulence and explosions are still important for the process of star formation because they control the birth correlations in space and time. Most star formation appears triggered by some specific process, whether it is a galactic spiral shock, the expansion of a superbubble, the compression of a bright-rimmed globule, or some seemingly random compressive event in a supersonically turbulent flow. These processes give space and time sequences for star birth that are well observed. Many examples were given at this conference. Shocks are the link between large-scale but weak galactic processes and small-scale but strong final collapses. The rate limiting step is on the largest scale, where the dynamical time is slowest. Both gravitational instabilities and pressurized triggering seem to work on the same local dynamical time, making it difficult to tell that star formation is highly triggered when observing only galactic scales.
\end{abstract}

Keywords. turbulence, instabilities, shock waves, stars:formation, ISM: structure

\section{Introduction}

Instabilities, explosions, galaxy interactions, and other dynamical processes all produce relative motions that can exceed the local thermal sound speed. If the momentum transferred by these motions through a galaxy cannot be quickly damped, then they should generate turbulence. This damping condition is essentially the same as the Reynolds number condition, i.e., that forced motions lead to turbulence when the Reynolds number is large (the Reynolds number is the ratio of the mixing velocity multiplied by the mixing scale to the viscous coefficient). In galaxies, the primary motions are supersonic and much of the damping is in shocks.

We have seen many talks at this conference showing how interstellar turbulence might be generated. Each model was able to highlight certain specific processes to the exclusion of others and then study the results. This method is less confusing than observing the real ISM, which combines them all. As a result, we have a good picture for the origin of ISM motions, although the relative importance of various processes in different regions is still uncertain.

The primary question addressed at this conference is why processes like ISM turbulence and expansional motions from HII regions, supernovae, and stellar winds are relevant to star formation. These processes would seem to be disruptive rather than collective, whereas the collection of gas into dense clouds is the first step toward star formation. Observations of actual star birth, consisting of gravitational collapse to a disk and the emergence of a protostar, do not reveal much about ISM turbulence and triggering. The boundary conditions for star formation do, however. Turbulence and triggering show up in various space and time correlations surrounding the star birth event (Sect. 2). They do not seem to influence the area-average star formation rate, though. 
We might guess that the star formation rate (in $\mathrm{M}_{\odot} \mathrm{pc}^{-3} \mathrm{Myr}^{-1}$ ) in some region of average density $\rho$ scales with $\epsilon \rho(G \rho)^{1 / 2}$, where $\epsilon$ is the fraction of the gas that is converted into stars at the average dynamical rate (which is $[G \rho]^{1 / 2}$ ). If star formation occurs in $D$ dynamical times, then $\epsilon$ equals $1 / D$ times the overall efficiency of star formation in that region. This expression for the star formation rate should apply if the observation of a particular molecule is sensitive to a wide range of intrinsic densities, so both the clump and the interclump gas are observed. CO is like this for molecular clouds at normal pressures in normal galaxies (not dwarf Irregulars). Then the molecule traces the total mass, and the observed mass per unit volume is the average density used in this expression. If a molecule traces only dense clumps, however, then the total emitting mass of the molecule divided by the cloud volume is not the average density, but less. Also in this case, the mass above the threshold for detection increases as the average density of the cloud increases. Then the star formation rate per unit core mass would be more constant than it is for $\mathrm{CO}$; it could be relatively independent of the average density detected by that molecule. This difference between diverse-density tracers and high-density tracers could partially explain the difference between the 1.5 power law for scaling with $\mathrm{HI}+\mathrm{CO}$ column density (Kennicutt 1989) and the 1 power law for scaling with HCN (Gao \& Solomon 2004). In addition, the HCN molecule probably traces starforming clumps which are already evolving toward stars as fast as they can with a certain efficiency. Then the star formation rate scales only with the average density of this molecule. Lower-density tracers like CO observe the gas before it reaches this stage, so there has to be an extra factor that determines the rate at which CO turns into dense clumps; this is the role of the $(G \rho)^{1 / 2}$ factor for average density $\rho$ determined from CO.

A local star formation rate per unit area equal to $A \Sigma^{1.5}$ also gives a global star formation rate per unit area proportional to the average $\langle\Sigma\rangle^{1.5}$, which may be written as $B\langle\Sigma\rangle^{1.5}$ for $B \sim A$. This can be seen from the integral over the disk of the local rate, using an exponential disk $\Sigma(r)=\Sigma_{0} \exp \left(-r / r_{D}\right)$. The integrated rate is

$$
\frac{\int_{0}^{R} A \Sigma_{0}^{1.5} \exp \left(-1.5 r / r_{D}\right) 2 \pi r d r}{\pi R^{2}}=\frac{2 A r_{D}^{2} \sigma_{0}^{1.5}}{1.5^{2} R^{2}}
$$

where small terms $\exp \left(-1.5 R / r_{d}\right)$ have been dropped in comparison to 1 . The average column density is $\langle\Sigma\rangle=\int_{0}^{R} \Sigma(r) 2 \pi r d r /\left(\pi R^{2}\right)=2 r_{D}^{2} \Sigma_{0} / R^{2}$. Writing the star formation rate as $B\langle\Sigma\rangle^{1.5}$ then gives

$$
B=\frac{2 A R}{2^{1.5} 1.5^{2} r_{D}}=0.3 \frac{A R}{r_{D}} \sim A
$$

because $R / r_{D} \sim 3-4$ for Freeman disks. Thus the global Kennicutt (1989) law is also consistent with a local relation of this type, as expected from theory (Elmegreen 2002).

The final efficiency of star formation depends on the density. It is $\sim 5 \%$ for OB associations and up to $\sim 30 \%$ for the cores of OB associations where clusters form. On a galactic scale, $\epsilon \sim 1.2 \%$ from the rotation rate correlation in Kennicutt (1998), considering that the average density in a galaxy is about the critical tidal density. In M33, $\epsilon \sim 60 \%$ if only the CO-emitting gas is used for $\rho$ (from data in Heyer et al. 2004, as derived in Elmegreen 2005). The increase for the CO coefficient is because the CO-emitting gas is a small fraction of the total.

We can get pretty far in predicting the star formation rate using only the density. This suggests that star formation is controlled primarily by gas self-gravity. In that case, why should we be concerned with sequential triggering and turbulence? The answer to this question lies entirely in the observed correlations. 


\section{It's the correlations...}

Star formation is linked to sequential triggering processes because of observed space and time sequences where young stars appear in the bright rims of OB associations, and in swept-up shells and spiral density waves. Early models of triggering were for different reasons. Opik (1953) suggested that supernovae trigger star formation in order to explain the expansion of OB associations, while Oort (1954) suggested that HII region pressures do the same thing. There may be some component of the expansion from triggering processes, but generally the density of a giant molecular cloud is so high that supernovae and HII regions do not accelerate the molecular gas much. Stellar expansion should come from gas dispersal out of clusters, a proposal first made by Zwicky (1953), and from runaway stars. In fact, most of the large expansion observed by Blaauw (1952) is the result of a few runaway O-type stars.

The more subtle correlations that suggest triggering, namely the spatial and temporal sequences of subgroups in OB associations, were first systematically described by Blaauw (1964). Blaauw's review paper effectively began the modern study of triggered star formation, although the real beginning had to wait a decade for the discovery of molecular clouds. Today, triggering of this type is commonly revealed by molecular cloud regions that contain young stars inside swept-up layers and bright rims. A previous generation of stars made these disturbances through some combination of stellar winds, ionization, and supernovae. Many talks and posters at this conference have illustrated shells, bright rims, and globules in which star formation has been triggered by compression from an older generation of stars.

Similarly for turbulence triggering, the importance of turbulence is revealed through space and time correlations in young stars that are reminiscent of those in turbulent fluids. For example, there is hierarchical structure in young stellar groupings (Scalo 1985; Feitzinger \& Galinski 1987; see Elmegreen et al. 2006 and references therein) that ranges from flocculent spiral arms on large scales to star complexes (Efremov 1995), OB associations, OB subgroups, and dense cores on small scales. There are also power-law size, luminosity, and mass distributions for stellar groupings, clusters, and star-forming clouds that reflect the scale-free conditions of turbulent flows. The optical light in galaxies also has a power-law power spectrum similar to that of the gas, partly because young stars cluster together like the gas and partly because of extinction effects (Elmegreen et al. 2003; Willett et al. 2005). Star clusters also group together with a power law autocorrelation function (Zhang et al. 2001).

Temporal correlations for turbulence triggering show up in comparisons between the duration of star formation and the size of the region: bigger regions form stars longer, in proportion to the square root of their size (Efremov \& Elmegreen 1998). This is like the size-linewidth relationship if we convert the ratio between size and linewidth to a turbulent crossing time. The observation is different than the correlation expected if star formation "spreads like a disease" (Baade 1963). In that case, the duration should scale with the square of the size, as in a random walk. It is also different from that expected from shear, in which case the relation should be linear (the correlation was found in the LMC where there is no shear). The result makes sense locally: Gould's Belt size regions and star complexes $\sim 300 \mathrm{pc}$ in size form stars for $\sim 50 \mathrm{Myr}, \mathrm{OB}$ associations $\sim 30 \mathrm{pc}$ in size form stars for $\sim 15 \mathrm{My}$, OB subgroups and GMC cores $\sim 3$ pc in size form stars for $\sim 5 \mathrm{My}$, and so on, with the square root dependence. The startling point about this is that a wide range of scales satisfies the correlation, which means that each scale has a duration of star formation proportional to the local crossing time of ISM turbulence on that scale. The large and small scale processes are similar in their dynamics. 


\section{If there is a sensitivity to $\epsilon \rho(G \rho)^{1 / 2}$ and $Q$, then how can there also be sequential \& turbulence triggering?}

A star formation rate proportional to $\epsilon \rho(G \rho)^{1 / 2}$ and the $Q$ threshold for star formation suggest that self-gravitational instabilities initiate star formation on a galactic scale and that gravity-dominated processes, like collapse or retarded collapse, control the rate. These relations do not seem to fit in with star formation that has spatial and time correlations reminiscent of sequential triggering and turbulence triggering. How can all three processes operate at the same time? Are the triggering mechanisms insignificant compared to self-gravitational instabilities? Or do the correlated processes have the same threshold and Schmidt law?

ISM instabilities and stellar compressions all operate at pressures much less than the pressure of a final pre-collapse star-forming clump. The instabilities sensitive to $Q$ begin on the scale of the ambient Jeans length, which is several $\mathrm{kpc}$ in a typical spiral galaxy disk, and primarily produce flocculent spiral arms, which shear away and disperse in $\sim 100 \mathrm{Myr}$. This is a relatively mild disturbance on average. A giant shell is also relatively mild compared to the pressure of a star-forming clump. The high pressure phases of all stellar-type explosions and expansions occupy only a small fraction of the ISM volume not enough to influence much star formation by direct compression of random clouds (Elmegreen 2004). Jenkins \& Tripp (2001) have shown, for example, that most regions have pressures within only a factor of $\sim 3$ times the average; $1 \%$ of the mass has a pressure 30 times the average. Still, what begins as a mild ISM disturbance can end up as a network of high-pressure star-forming cores because of the further action taken by turbulence. Turbulence, and the expansional motions that lead to swept-up shells and layers, have the property that they concentrate low-pressure, high-volume energy (e.g., flocculent arms and supernova cavities) into high-pressure, low-volume forms, such as shocks, multiple shocks (converging or intersecting), and vortices. In these high-pressure forms, enhanced magnetic diffusion, enhanced thermal cooling, and enhanced kinetic energy dissipation (also via shocks) remove supportive energy and let local self-gravity take over. Then the local pressure can go up enormously as a dense core forms. Star formation soon follows.

Shocks are the link between galactic scale processes (which are big but intrinsically weak) and final star formation processes (which are small but intrinsically strong). Freefall collapse (Hoyle 1953) is not the link because magnetic fields retard this collapse, random motions divert it, and density gradients stretch it. And while turbulence and compression help convert low density gas into high density gas, they also set up the spatial and temporal correlations that appear in young star fields without affecting the overall star formation rate much, which is still limited on the large-scale by self gravitational dynamics. The rate limiting process for star formation is on the largest scale, where the density is lowest and the timescale is longest. If intermediate scales are too slow to keep up the pace dictated by the large scale gravitational instabilities, then they will produce a bottleneck, changing the distribution of density so that more material at the bottleneck compensates for the relatively low rate there. But the overall star formation rate should not change. This means that the galactic-scale star formation rate is not controlled by the rate at which tiny solar-mass clumps collapse into stars. The rate is determined at the start of the whole process, on a large scale.

To put this another way, the star formation rates, but not the correlations, are produced by ambient disk gravity, and the correlations, but not the star formation rates are produced by the intermediate-scale processes (turbulence, shells) which channel the low-P, high-V perturbations down to high-P, low-V structures in which final collapse 
begins. In an analogy my former-teenagers would have understood: $Q$ is the green light, $\epsilon \rho(G \rho)^{1 / 2}$ is the speed limit, and the correlations are the road patterns.

The Schmidt law and Toomre threshold also apply somewhat directly to sequential and turbulence triggering, which have similar average rates and thresholds. The triggering time for star formation in a swept-up shell is about equal to the dynamical time in the surrounding medium, $0.3\left(4 \pi G \rho_{0}\right)^{-1 / 2}$, and the condition that collapse occurs before the shell shears away is about the same as the Toomre $Q<1$ condition (Elmegreen, Palouš \& Ehlerova 2002). Turbulence triggering also has its rate-determining step on the largest scale, and the compression time over a scale height is about the dynamical time in the disk (by definition of the scale height). Also, turbulence compression requires that the random motions be able to move for at least a scale height in order to build up enough mass to be gravitationally unstable at the ambient rms velocity dispersion. These motions are influenced by Coriolis forces, so the condition that the epicyclic radius for the random motions exceeds the gaseous scale height is again $Q<1$.

\section{The efficiency}

Turbulence and stellar pressures affect the efficiency $\epsilon$ somewhat. In a turbulent star formation model, $\epsilon$ is related to the mass fraction of gas in a dense form (i.e., in the tail of the log normal pdf - Elmegreen 2002; Kravtsov 2003; Krumholz \& McKee 2005; for applications to the IMF, see Padoan \& Nordlund 2002). At higher Mach numbers and higher mean densities, the tail fraction exceeding a certain critical density is higher, so the efficiency should be higher. In a sequentially triggered model, $\epsilon$ is proportional to the number of generations of star formation in a cloud. Star formation, can, in principle, continue in a sequentially triggered fashion, generation after generation, until most of the gas is used up. In both cases, a lot of the microphysics is in $\epsilon$, while the macrophysics is still galactic-scale self-gravity.

\section{The even bigger picture}

When star formation is regulated by the Toomre parameter $Q$, the gas mass tends to stay comparable to the threshold value (Zasov \& Smirnova 2006) and the star formation rate is, on average, equal to the average ISM accretion rate. This is because $Q$ selfregulates via gas consumption and perhaps massive-star feedback to remain at a value near 1 on a Gyr timescale (Fuchs \& von Linden 1998; Bertin \& Lodato 2001). Accretion and mass-redistribution inside a galaxy, from spiral and bar torques, for example, determine the details of the density and column density profiles.

When the Sun formed (at redshift $z=0.45$ ), the mass column density from stars in the Milky Way was only half what it is today. The disk was smaller and more fragile. The star formation rate has apparently not changed much since then, aside from factor-of-5 variations either way, but star formation is still winding down universally. The "red and dead" Hubble types, i.e., the ellipticals, S0's and Sa's, are creeping toward the Milky Way's Hubble type, which is Sbc. Still we have a long way to go. The LMC stream and even the whole LMC and SMC will eventually accrete to the Milky Way. Such accretion increases $\Sigma / \Sigma_{\text {crit }}$ locally, and then star formation proceeds at the usual dynamical rate.

\section{References}

Baade, W. 1963, in: C. Payne-Gaposchkin (ed.), The Evolution of Stars and Galaxies (Cambridge: Harvard University Press), Chapter 16 
Bertin, G. \& Lodato, G. 2001, A\& $A$ 370, 342

Blaauw, A. 1952, BAN 11, 405

Blaauw, A. 1964, ARAA 2, 213

Efremov, Yu. N. 1995, AJ 110, 2757

Efremov, Y.N. \& Elmegreen, B.G. 1998, MNRAS 299, 588

Elmegreen, B.G. 2002, ApJ 577, 206

Elmegreen, B.G., Leitner, S.N., Elmegreen, D.M. \& Cuillandre, J.-C. 2003, ApJ 593, 333

Elmegreen, B.G., Palouš, J. \& Ehlerova, S. 2002, MNRAS 334, 693

Elmegreen, B.G. 2004, in: H.J.G.L.M. Lamers, L.J. Smith \& A. Nota (eds.), The Formation and Evolution of Massive Young Star Clusters (ASP-CS), 322, 277

Elmegreen, B.G. 2005, in: Jose Carlos del Toro Iniesta, et al. (eds.), The many scales in the Universe - JENAM 2004 Astrophysics Reviews (Dordrecht: Kluwer), p. 99

Elmegreen, B.G., Elmegreen, D.M., Chandar, R., Whitmore, B., \& Regan, M. 2006, ApJ 644, 879

Feitzinger, J.V. \& Galinski, T. 1987, A $\& A$ 179, 249

Fuchs, B. \& von Linden, S. 1998, MNRAS 294, 513

Gao, Y. \& Solomon, P.M. 2004, ApJ 606, 271

Heyer, M.H., Corbelli, E., Schneider, S.E., \& Young, J.S. 2004, ApJ 602, 723

Hoyle, F. 1953, ApJ 118, 513

Jenkins, E.B \& Tripp, T.M. 2001, ApJS 137, 297

Kennicutt, R.C. 1989, ApJ 344, 685

Kravtsov, A.V. 2003, ApJ 590, L1

Krumholz, M.R. \& McKee, C.F. 2005, ApJ 630, 250

Oort, J.H. 1954, BAN 12, 177

Opik, E.J. 1953, Irish J. Astron 2, 219

Padoan, P. \& Nordlund, A. 2002, ApJ 576, 870

Scalo, J. 1985, in: D.C. Black, \& M.S. Matthews (eds.), Protostars and Planets II (Tucson: Univ. of Arizona), p. 201

Willett, K.W., Elmegreen, B.G. \& Hunter, D.A. 2005, AJ 129, 2186

Zasov, A.V. \& Smirnova, A.A. 2006, AstL 31, 160

Zhang, Q., Fall, S.M. \& Whitmore, B.C. 2001, ApJ 561, 727

Zwicky, F. 1953, PASP 65, 205 